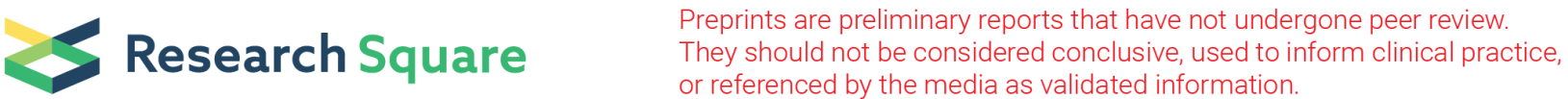 Atrial myxoma with renal colic
}

\section{Yang Guo}

Sichuan Provincial People's Hospital: Sichuan Academy of Medical Sciences and Sichuan People's Hospital

\section{Min Cao}

Sichuan Provincial People's Hospital: Sichuan Academy of Medical Sciences and Sichuan People's Hospital

\section{Xiaobo Huang}

Sichuan Provincial People's Hospital: Sichuan Academy of Medical Sciences and Sichuan People's Hospital

\section{Lingai Pan ( $\nabla$ winter-sun-12@outlook.com )}

Sichuan Provincial People's Hospital: Sichuan Academy of Medical Sciences and Sichuan People's Hospital

\section{Case report}

Keywords: myxoma, renal colic, embolization, case report

Posted Date: March 26th, 2021

DOl: https://doi.org/10.21203/rs.3.rs-284770/v1

License: (9) (1) This work is licensed under a Creative Commons Attribution 4.0 International License. Read Full License 


\section{Abstract}

\section{Background}

cardiac myxoma is a common benign tumor of the heart. It can have any presentation such as mitral stenosis, embolization. The best treatment is surgical resection.

\section{Case presentation}

A 30-year-old man presented with 2-days of right renal colic and chest tightness, dyspnea. He was diagnosed as renal infarction through ultrasound in another hospital and received some analgesic drugs. But the symptoms didn't resolved and he came to our ICU(Intensive Care Medicine). We performed the ultrasonography and found a mass in the right atrium computed tomography angiography cofirmed it, and it was cross-valve growth, irregular shape, and slight enhancement of the enhanced edge, consider myxoma. Multiple non-reinforced areas were seen in the right renal cortex and medulla, right renal artery embolization, right renal infarction might be considered. The patient was taken to the operating room for emergent myxoma resection, renal artery thrombectomy.

\section{Conclusion}

Myxomas should be in differential diagnosis in patient present with thromboembolism. Early diagnosis and surgical are crucial treatment in deal with myxoma.

\section{Background}

Atrial myxoma is the most common benign cardiac tumor representing $50-75 \%$ of all cardiac tumors ${ }^{[1]}$.The gold standard treatment is surgical excision if symptomatic ${ }^{[2]}$.Cardiac biopsy is not usually needed, but if malignancy is suspected, then the biopsy should be done after surgical excision ${ }^{[3]}$.Systemic embolism due to atrial myxoma has been well reported, especially embolic stroke ${ }^{[4]}$. However, renal colic are rarely associated to atrial myxoma. We present the case of a man presented with 2-days of right renal colic and chest tightness, dyspnea and do ultrasonography and computed tomography angiography (CTA) immediately .

\section{Case Presentation}

A 30-year-old man with 2-days of right renal colic and chest tightness, dyspnea. There were no fever, chest pain, headache. He was diagnosed as renal infarction through ultrasound in another hospital and received some analgesic drugs. But the symptoms didn't resolved and he came to our ICU. On physical examination, he was in acute distress due to pain in right renal. Lungs were clear to auscultation and cardiac examination revealed enlargement of the heart, with a diastolic murmur. Electrocardiogram showed normal sinus rhythm. Abdomen examination showed right kidney tenderness. Laboratory 
investigations were significant for leukocytosis of $21.76 * 109 / \mathrm{L}$ and normal level of troponin. Routine urine test showed red blood cell 115.2/ul $(<11.5 / \mathrm{ul})$.

We performed the ultrasonography and found a mass in the right atrium (Figure1). Computed tomography angiography was done immediately, which demonstrated that a patch-like low-density shadow was seen in the left atrium and left ventricle (Figure2), and it was cross-valve growth, irregular shape, and slight enhancement of the enhanced edge, consider myxoma. Multiple non-reinforced areas were seen in the right renal cortex and medulla, right renal artery embolization, right renal infarction might be considered (Figure3).

The patient was taken to the operating room for emergent myxoma resection, renal artery thrombectomy. The size of left atrial myxoma was $76 \mathrm{~mm} \star 50 \mathrm{~mm} \star 44 \mathrm{~mm}$ (Figure4a), the two myxoma emboli in the right renal artery were $3 \mathrm{~mm} \star 3 \mathrm{~mm}$ and $2 \mathrm{~mm} * 1 \mathrm{~mm}$ (Figures $4 \mathrm{~b}$ ), and there was a massive ischemic area in the right kidney. Pathology report subsequently confirmed the diagnosis of myxoma. He recovered well from surgery.And he will will follow the doctor's outpatient follow-up cardiac ultrasound and related examinations.

\section{Discussion And Conclusion}

The incidence of the renal colic related to an atrial myxoma is seldom appearance, and there are no guidelines to guide treatment. The patient presented with 2-days of right renal colic and chest tightness, dyspnea. Ultrasonography and computed tomography angiography (CTA) immediately after admission, clearly demonstrating a a patch-like low-density shadow in the left atrium and left ventricle, as one of the pathognomonic signs of cardiac myxoma. This rare and unexpected, but clearly find the cause of disease-cardiac myxoma shedding. The operation is very timely, because the right kidney has formed a huge ischemic area. So, early diagnosis and surgical are crucial treatment in deal with myxoma. And long term follow-up is recommended. Outcome of most patients is excellent.

Myxomas should be in differential diagnosis in patient present with thromboembolism. And early diagnosis and surgery are essential for patients.

\section{Patient Perspective}

The patient believed that the treatment he received was very timely-the atrial myxoma was discovered in time, and the embolus was removed by surgery in time, which saved the kidney from ischemic necrosis.

\section{Declarations}

\section{Acknowledgments}

Not applicable. 


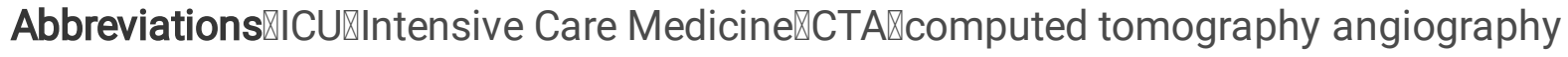

\section{Authors' contributions}

The conception and design of the study:Lingai Pan,Min Cao. Contributors Yang Guo and Xiaobo Huang were responsible for the data collection.Drafting the article: Min Cao. All authors contributed to writing the manuscript and approved the final manuscript.

Funding: The authors have not received funding for the preparation of the present manuscript.

Availability of data and materials $₫$ The datasets used and/or analyzed during the current study are available from the corresponding author on reasonable request.

Ethics approval and consent to participate $\triangle T$ The patient provided consent for the publication of her case including any individual details and images.

Consent for publication $\nabla$ The patient provided consent for the publication of her case including any individual details and images.

Competing interests $\varangle$ The authors declare that they have no conflicts of interest and competing interests.

\section{References}

1. Acampa M, Guideri F, Tassi R, D'Andrea P, Marotta G, Lo Giudice G, Martini G. Thrombolytic treatment of cardiac myxoma-induced ischemic stroke: a review. Curr Drug Saf. 2014;9:83-8.

2. Yüksel A, Saba D, Velioğlu Y, Ener S, Özkan H. Biatrial approach provides better outcomes in the surgical treatment of cardiac myxomas. Braz J Cardiovasc Surg. 2016;31:309-17. 10.5935/16789741.20160066.

3. Yuan SM, Yan SL, Wu N. Unusual aspects of cardiac myxoma. Anatol J Cardiol. 2017;17:241-7. 10.14744/AnatolJCardiol.2017.7557.

4. Lee VH, Connolly HM, Brown RD. Jr Central nervous system manifestations of cardiac myxoma. Arch Neurol. 2007;64(8):1115-20.

\section{Figures}




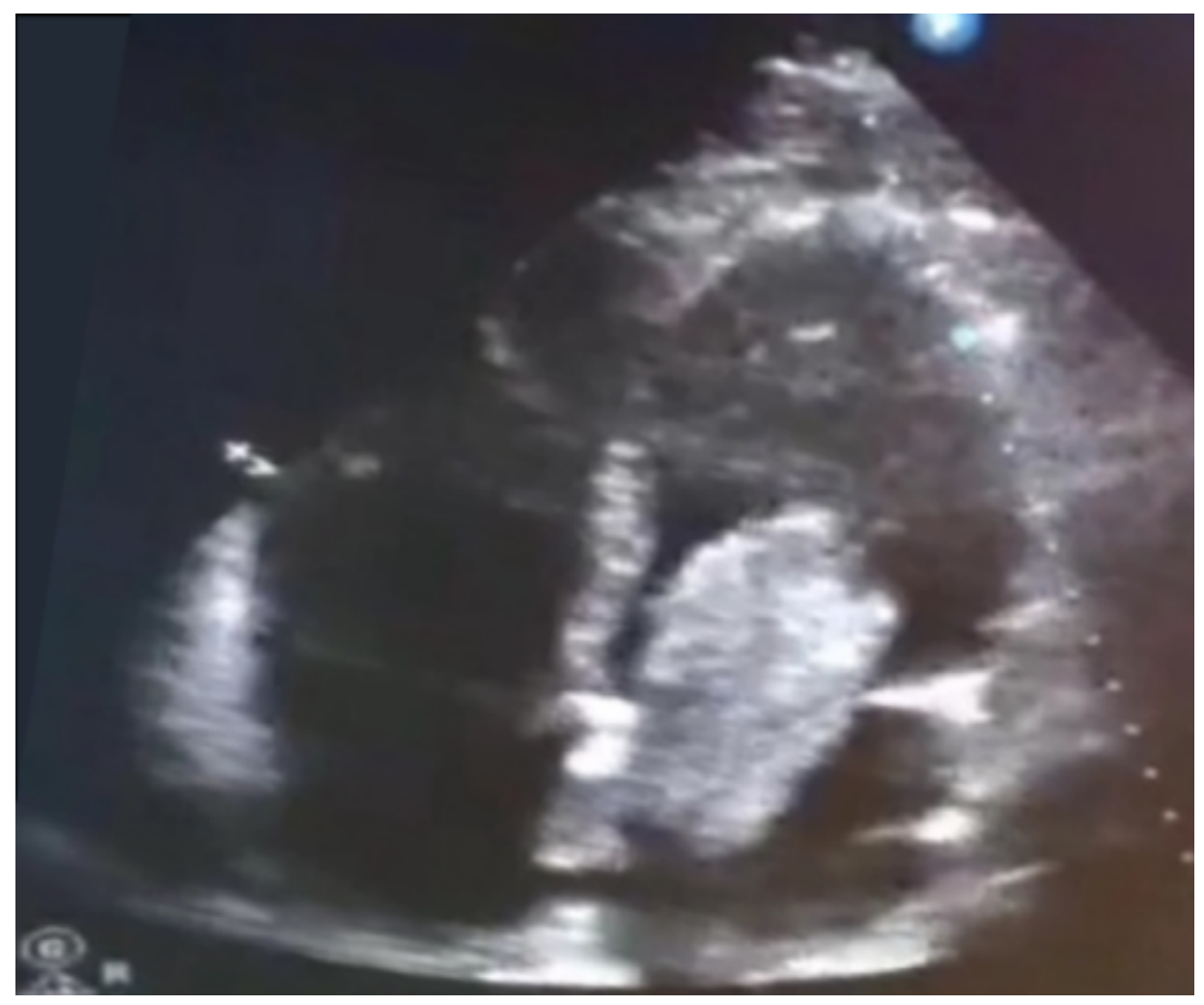

Figure 1

Transthoracic echocardiography showing left atrial mass consistent with myxoma and cross-valve growth.

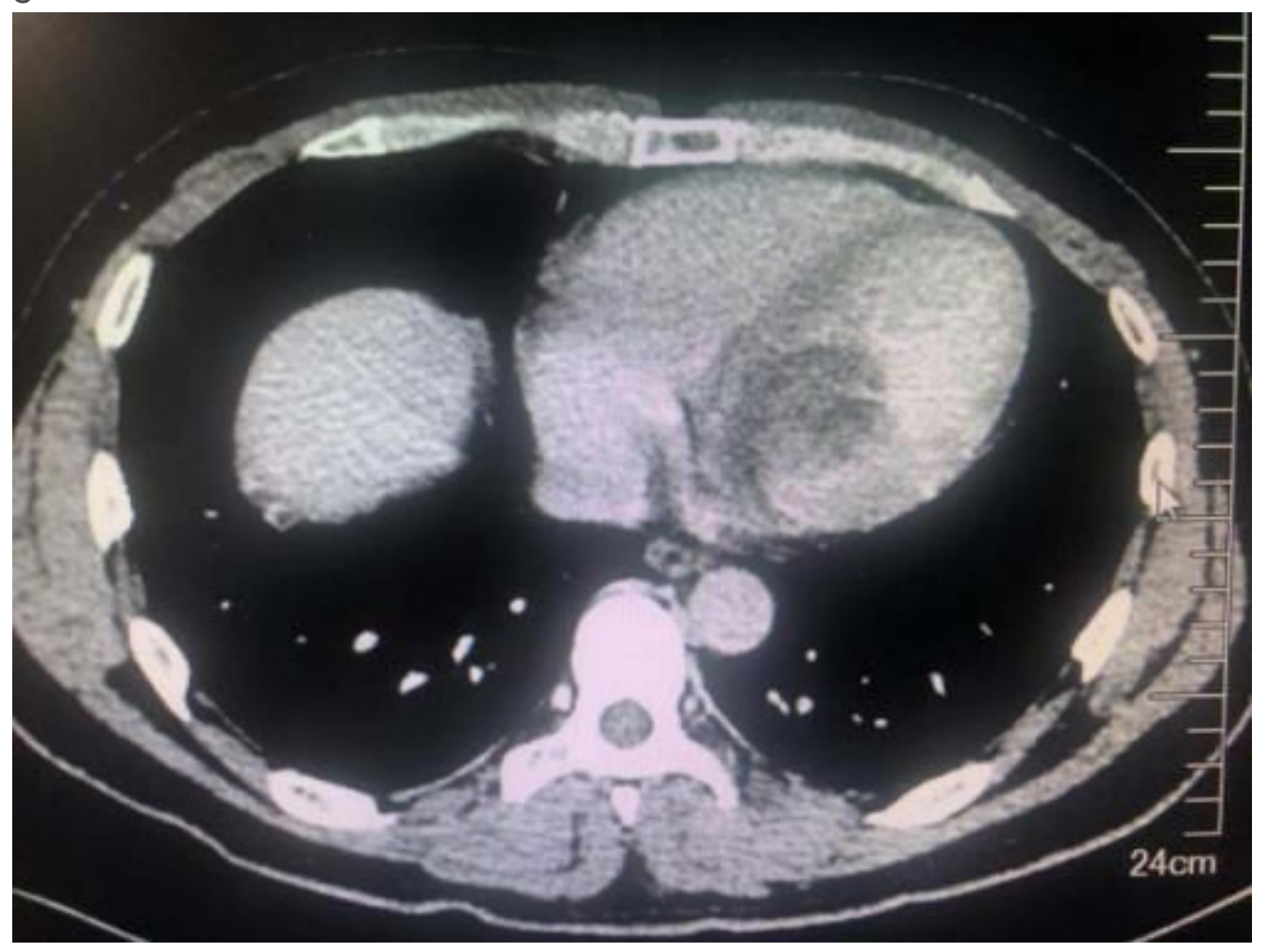

Figure 2 
Computed tomography angiography demonstrated a patch-like low-density shadow was seen in the left atrium and left ventricle.

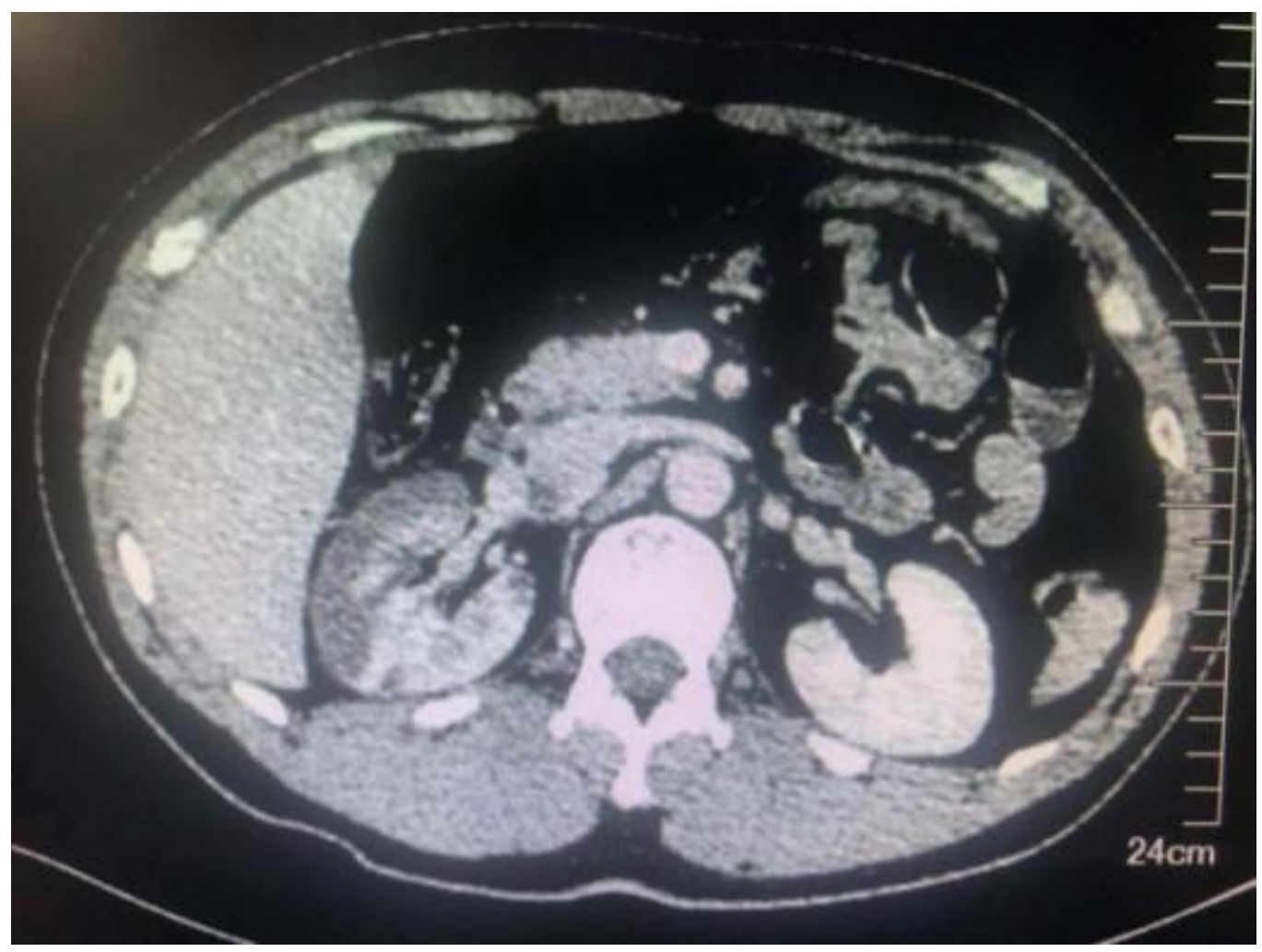

\section{Figure 3}

Multiple non-reinforced areas were seen in the right renal cortex and medulla, right renal artery embolization, right renal infarction.

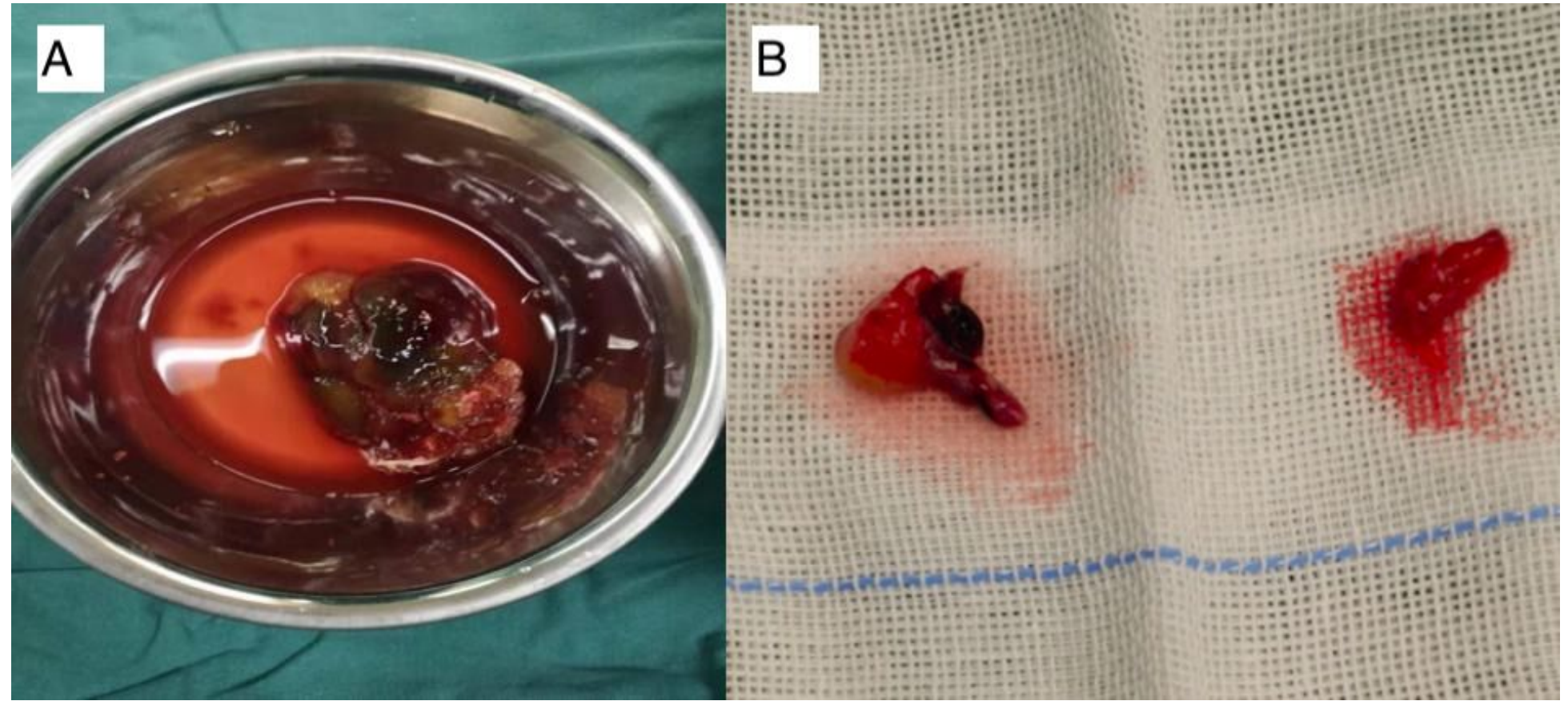

Figure 4 
a Left atrial myxoma was $76 \mathrm{~mm} \star 50 \mathrm{~mm} * 44 \mathrm{~mm}$ (left). b Myxoma emboli in the right renal artery were $3 \mathrm{~mm} \star 3 \mathrm{~mm}, 2 \mathrm{~mm} \star 1 \mathrm{~mm}$ respectively (right).

\section{Supplementary Files}

This is a list of supplementary files associated with this preprint. Click to download.

- CAREchecklistEnglish2013.pdf 